Article

\title{
A Comprehensive Signal-Based Fault Diagnosis Method for Lithium-Ion Batteries in Electric Vehicles
}

\author{
Xinwei Cong ${ }^{1}\left(\mathbb{D}\right.$, Caiping Zhang ${ }^{1, *}$, Jiuchun Jiang ${ }^{2,3}$, Weige Zhang ${ }^{1}$, Yan Jiang ${ }^{2,4}$ and Linjing Zhang ${ }^{1}$ \\ 1 National Active Distribution Network Technology Research Center (NANTEC), Beijing Jiaotong University, \\ Beijing 100044, China; 15117399@bjtu.edu.cn (X.C.); wgzhang@bjtu.edu.cn (W.Z.); lj.zhang@bjtu.edu.cn (L.Z.) \\ 2 Sunwoda Co., Ltd., Shenzhen 518100, China; jiangjc@sunwoda.com (J.J.); jiangyan3@sunwoda.com (Y.J.) \\ 3 School of Electrical and Electronic Engineering, Hubei University of Technology, Wuhan 432200, China \\ 4 School of Electric Power, South China University of Technology, Guangzhou 510006, China \\ * Correspondence: zhangcaiping@bjtu.edu.cn
}

Citation: Cong, X.; Zhang, C.; Jiang, J.; Zhang, W.; Jiang, Y.; Zhang, L. A Comprehensive Signal-Based Fault Diagnosis Method for Lithium-Ion Batteries in Electric Vehicles. Energies 2021, 14, 1221. https://doi.org/ $10.3390 /$ en14051221

Academic Editor: Peter Foot

Received: 2 February 2021

Accepted: 20 February 2021

Published: 24 February 2021

Publisher's Note: MDPI stays neutral with regard to jurisdictional claims in published maps and institutional affiliations.

Copyright: (c) 2021 by the authors. Licensee MDPI, Basel, Switzerland. This article is an open access article distributed under the terms and conditions of the Creative Commons Attribution (CC BY) license (https:// creativecommons.org/licenses/by/ $4.0 /)$.
Abstract: To enhance the operational reliability and safety of electric vehicles (EVs), big data platforms for EV supervision are rapidly developing, which makes a large quantity of battery data available for fault diagnosis. Since fault types related to lithium-ion batteries play a dominant role, a comprehensive fault diagnosis method is proposed in this paper, in pursuit of an accurate early fault diagnosis method based on voltage signals from battery cells. The proposed method for battery fault diagnosis mainly includes three parts: variational mode decomposition in the signal analysis part to separate the inconsistency of cell states, critical representative signal feature extraction by using a generalized dimensionless indicator construction formula and effective anomaly detection by sparsity-based clustering. The signal features of the majority of signal-based battery fault detection studies are found to be particular cases with a specific set of parameter values of the proposed indicator construction formula. With the sensitivity and stability balanced by appropriate moving-window size selection, the proposed signal-based method is validated to be capable of earlier anomaly detection, false-alarm reduction, and anomalous performance identification, compared with traditional approaches, based on actual pre-fault operating data from three different situations.

Keywords: dimensionless indicator; fault diagnosis; lithium-ion battery; unsupervised learning; variational mode decomposition

\section{Introduction}

Lithium-ion batteries are usually used in the power supply and storage of electric vehicles (EVs) because of their superior performance [1]. With a number of favorable policies issued and implemented in recent years, the demand for reliability and safety of lithium-ion batteries is steadily on the increase. As a consequence, potential battery abnormality and faults require accurate detection, isolation, and identification as early as possible, so that fault-tolerant operation for minimizing the battery performance degradation can be implemented in time to avoid dangerous situations and battery failure [2,3]. Generally, battery fault diagnosis methods include the following categories: model-based, data-driven, knowledge-based, and signal-based methods [4], and fault types of EV battery system consist of battery faults [5-7] (such as overcharging, overdischarging, overheating, loose connections, insulation failure, state inconsistency, external/internal short circuits, electrolyte leakage, swelling, accelerated degradation and thermal runaway), sensor faults, and actuator faults [8].

As for the model-based methods for lithium-ion batteries, by utilizing filters and observers (such as Kalman filter-based methods [7-10], particle filter [11], Lunberger observer [12], and adaptive observers [13]), the equivalent circuit model [14], the electrochemical model [6], and other models [15] at battery cell level are applied to trace 
and update parameters related to battery states, with excellent real-time monitoring performance [16]. However, the parameters are still challenging to be accurately estimated by signals collected in actual EV operation within a short period. Besides, a large quantity of studies on fractional order models (FOMs) along with their derivatives [17,18], and models on battery pack level [19-21] have been dedicated to enhance the accuracy of depicting complicated electrochemical behaviors, material properties, constructions, and the nonlinear fading mechanism of lithium-ion batteries [22]. But on account of almost open-loop estimation process, insufficient adaptivity makes it dissatisfactory to employ model-based methods in most practical cases. Moreover, the computational cost required is often not affordable by current BMS hardware [23] and potential failure in convergence under several working conditions [24]. Even under relatively ideal working conditions, the collected signals in a battery pack could still be influenced by the differences in the state of charge (SOC), state of health (SOH), internal impedance, and other external environmental factors, so it is necessary to make an effort to effectively distinguish various actual faults from severe inconsistency [19]. A further reason that makes it unfeasible to adopt model-based methods for time-efficient battery fault diagnosis in practice is that, their ideal boundary conditions are hardly satisfied in actual operation of EVs.

As for the non-model-based methods for battery fault diagnosis, on-board sensors are utilized to monitor various physical signals such as thermal, electrical, mechanical, etc., and the records are uploaded to the data platform after real-time processing by the battery management system (BMS). As one of the most fundamental signals to analyze, time series of cell voltage values have been typically adopted to realize efficient battery fault diagnosis in extensive studies, based on voltage and voltage difference thresholds [25], machine learning techniques [26], local outliers, the correlation [27], the information entropy [28], and their derivatives [29]. Without the requisite efforts in battery modeling, these methods are available for online applications and offline data analysis, but the performance is inevitably influenced by inconsistency of cell states and the quantity/quality of data records as well as measurement noise. The raw data containing the information to determine diversified states of the battery cells, are usually accompanied by precursory fault signals, noises brought by measuring facilities, and data loss during transmission and storage.

In brief:

(1) High computing capability for precise mechanism modeling and signal filtering is required, which is difficult for practical implementation of model-based fault diagnosis methods by current BMS hardware;

(2) Besides, corresponding information contained in data records related to some subtle precursory abnormal signals, and the impact of inconsistency on the fault detection should be separate away to enhance the robustness of the diagnosis method;

(3) Moreover, the adaptivity of the fault threshold should be considered to make the method reliable to different extents of abnormal performance observed in the sensor signals;

(4) Finally, considering the large environmental noise, inefficiently placed temperature sensors in reality, and the hysteresis characteristics of temperature signal records, electric signals are technically preferable in practical application, but traditional battery fault diagnosis methods based on voltage signals are hardly available to identify the detailed abnormal performance;

Considering all the challenging factors, a signal-based battery fault diagnosis method based on the voltage signals is proposed in the article. The overall flowchart is shown in Figure 1. The paper contains the following solutions in detail:

(1) Considering the excitations composed of various frequency components, some electrochemical behaviors can be prominently analyzed to illustrate the dynamic outputs of lithium-ion batteries mainly in the high frequency range, for there are small time constants of the response related to polarization, whereas the 
states of battery cells can be estimated by measuring in the low frequency range, as well as the diffusion process. Variational mode decomposition (VMD) algorithm is utilized to find out the components of voltage signals that are strongly associated with either the local response to external excitations, or long-term variation tendencies of battery states. In this way, corresponding functions will be realized on signal components in different frequency ranges. To our best knowledge, using VMD in signal analysis can attain some satisfying performance [30], compared with using other signal process methods such as empirical mode decomposition [23] that suffers from end effect and mode mixing, and wavelet decomposition [31] of which the wavelet basis becomes hard to determine, due to coupled performances and occurrence of different types of faults;

(2) Influenced by multiple external factors, it is hard to make robust judgments on abnormal signals by directly employing methods on raw signal data. Therefore, a generalized dimensionless indicator (GDI) extraction formula with moving-window observation is proposed to reduce the impact of the quality and the quantity of training data, and to effectively balance the sensitivity and stability of the signal features depending on the applied situation. Extensive interpretability of the GDI formula with reasonable physical significance will be validated within a list of effective signal features in existing literatures;

(3) As both short daily data and a considerable amount of historical data are likely to be used to determine a specific threshold, the clustering algorithm, as one of the unsupervised learning methods for effective anomaly detection, is utilized to find out the existing outliers in two kinds of sequences of proposed features extracted from a flexible number of training samples. The validation is made upon the battery pack data derived from the real-world thermal runaway cases, and the name of battery/EV manufacturers cannot be revealed due to their regulations related to data confidentiality, thus photos of BMS or burned ruins unavailable;

(4) To illustrate the feasibility of identifying some specific abnormal patterns in the moving-window observed voltage signals, three case studies with preferably selected parameters of the proposed formula are presented, and the time-priority weight is considered over multiple voltage segments in the quantified anomaly evaluation procedure for a definitive diagnosis report.

The remainder of this paper is organized as follows: Section 2 specifies the VMD algorithm and utilization, and GDI construction formula is introduced as the feature extraction method with moving-window observation in Section 3. Then the sparsity-based clustering method is introduced and implemented upon weighed feature sequences to obtain the diagnosis results in Section 4. The performance of the proposed method is verified, compared with several different methods in three practical cases in Section 5. At last, conclusions are presented with future work in Section 6. 


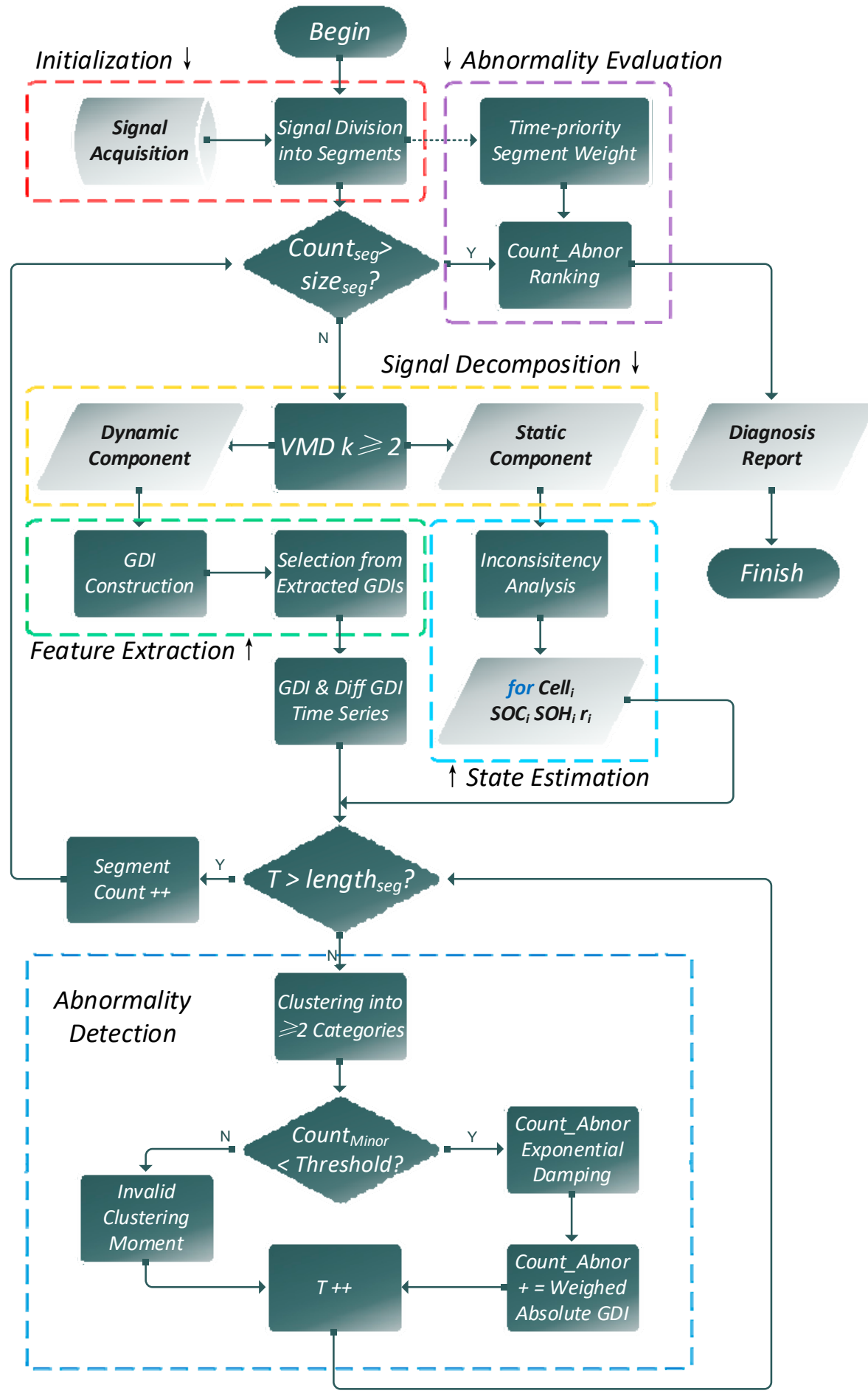

Figure 1. The overall flowchart of the proposed fault diagnosis method.

\section{Signal Processing with VMD}

The performance of using VMD for non-stationary and nonlinear signal analysis has been validated in extensive studies on mechanical vibration signals [32-35]. Enlightened by this outstanding signal processing technique, the assumption can be made that we can learn from machinery fault diagnosis to perform battery fault diagnosis, because the battery voltage signals share a lot of similarities with machinery vibration signals. Regarded as response signals carrying the information characterizing system states and potential faults, both of the two signals are observable outputs of sophisticated nonlinear systems 
with high uncertain excitations under actual operating circumstances. In order to acquire significant components for anomaly detection and fault diagnosis, this signal analysis method is introduced.

VMD was proposed by Dragomiretskiy and Zosso for adaptive and non-recursive signal analysis [36]. In order to distinguish the true fault signals from battery inconsistencies shown in voltage signals when an actual electric vehicle is operating, VMD is used to decompose voltage signals into narrow-band components with independent center frequencies. The decomposed battery signals are considered to contain two main components: the dynamic part, as the transient state response of actual vehicle operating without the offset caused by the battery state, and the static part, derived from varying battery states with the actual vehicle operating. The operation state of charging or discharging can be easily classified by current and speed in practice.

The framework of VMD is to evaluate the moderate bandwidth of $k$ number of decomposed components called intrinsic mode functions (IMFs), which are compact around independent center frequencies. In frequency spectrum, the cell voltage signals are initially processed by using the Hilbert transform, and then the formulated constrained variational optimization problem can be expressed by Equation (1):

$$
\begin{gathered}
\min _{\left\{u_{k}\right\}\left\{\omega_{k}\right\}}\left\{\sum_{k}\left\|\partial_{t}\left[\left(\delta(t)+\frac{j}{\pi t}\right) * u_{k}(t)\right] e^{-j \omega_{k} t}\right\|_{2}^{2}\right\} \\
\text { s.t. } \sum_{k} u_{k}=f
\end{gathered}
$$

where $u_{k}$ denotes the $k$-th component of the signal, $\omega_{k}$ denotes center frequency of the $k$-th component of the signal, $f$ denotes the original signal, $\delta$ denotes the Dirac distribution, and * denotes the convolution operator. After using a quadratic penalty term and Lagrangian multipliers, the constrained variational optimization problem can be further expressed by Equation (2):

$$
\begin{aligned}
L\left(\left\{u_{k}\right\},\left\{\omega_{k}\right\}, \lambda\right) & :=\alpha \sum_{k}\left\|\partial_{t}\left[\left(\delta(t)+\frac{j}{\pi t}\right) * u_{k}(t)\right] e^{-j \omega_{k} t}\right\|_{2}^{2} \\
& +\left\|f(t)-\sum_{k} u_{k}(t)\right\|_{2}^{2}+<\lambda(t), f(t)-\sum_{k} u_{k}(t)>
\end{aligned}
$$

where $\alpha$ denotes the balancing parameter of the data-fidelity constraint. Considering the actual sampling frequency and current ratio, the order of magnitude can be $10^{2}$ to $10^{3}$. Then it can be solved with the alternate direction method of multipliers, and all the IMFs gained from solutions in spectral domain of frequencies are shown by Equation (3):

$$
u_{k}^{n+1}(\omega)=\frac{f(\omega)-\sum_{i \neq k} u_{i}(\omega)+\frac{\lambda(\omega)}{2}}{1+2\left(\omega-\omega_{k}\right)^{2}}
$$

where $f(\omega), u_{i}(\omega), \lambda(\omega)$ and $u_{k}^{n+1}(\omega)$ respectively denote the Fourier transforms of $y(t)$, $y_{i}(t), \lambda(t)$ and $y_{k}^{n+1}(t)$ to be determined. The components in the time domain can be finally obtained as the real part from the inverse Fourier transform of the filtered signal.

Considering the specific problem of battery fault diagnosis, the decomposed IMFs with different main frequency bands will be further classified into two primary categories, for $k$ could be larger than 2, with the algorithm parameters determined by the actual sampling frequency. IMFs with lower frequency bands can efficiently express the variation of battery states with longer time scales, whereas components containing higher frequency bands can be loaded with potential transient features indicating certain abnormality as shown in Figure $2 \mathrm{~b}$. In this way, voltage data records are necessarily processed to reduce the impact of the uncorrelated state factors of cells. The features are then extracted and interpreted to fuse the evidence concerning the abnormalities in the following section. 
a) Cell voltage in case 1

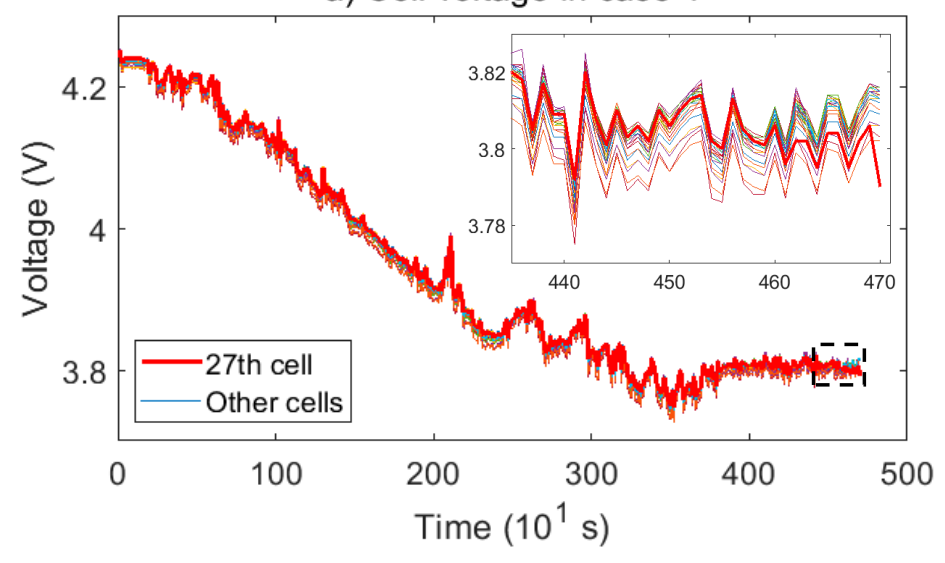

b) VMD result
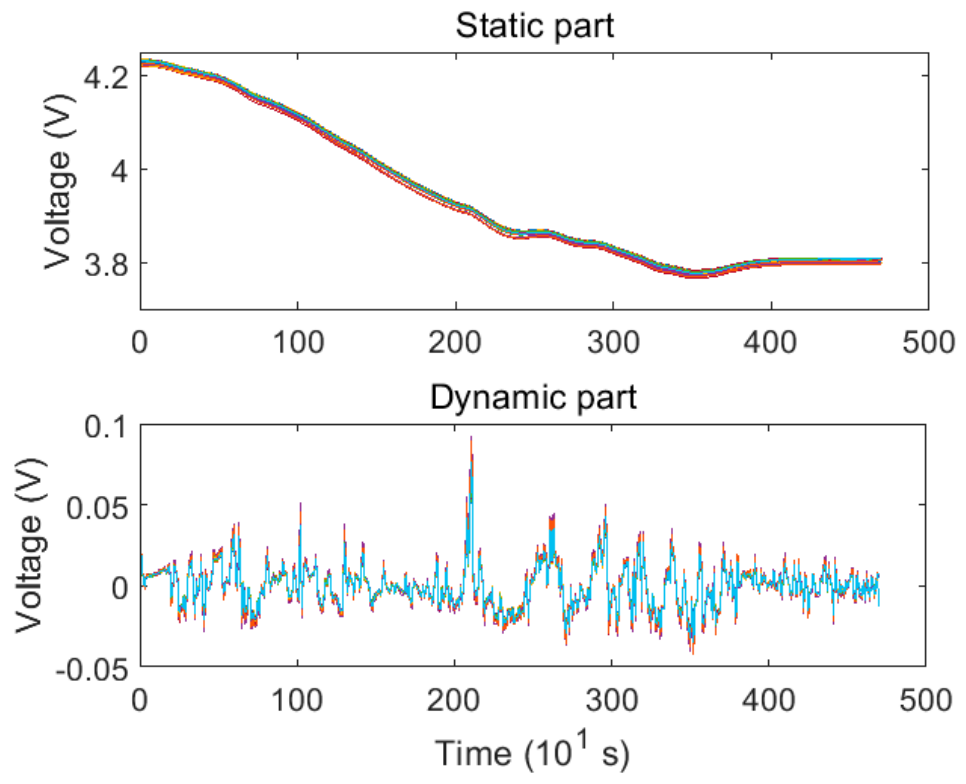

Figure 2. Voltage signal records in the first case, (a) Voltage signal records of 97 cells until the alarm of BMS (b) Decomposed voltage signal parts.

\section{Generalized Dimensionless Indicator Extraction Formula}

Dimensionless indicators (DIs) are diagnosis characteristics that are sensitive to battery abnormalities and faults, instead of working conditions, for they are less affected by the disturbance with the size of the moving window appropriately selected. In existing studies [37], DIs are traditionally defined using Equation (4):

$$
D I_{y}=\frac{\left[\int_{-\infty}^{+\infty}|y|^{l} p(y) d y\right]^{\frac{1}{T}}}{\left[\int_{-\infty}^{+\infty}|y|^{m} p(y) d y\right]^{\frac{1}{m}}}
$$

where $|y|$ denotes the signal amplitudes, $p(y)$ denotes the probability density function (PDF) of corresponding signal amplitudes, and $l, m$ are two different positive constants. If the quantity of signal records $N$ is sufficient in temporal moving window, and the weight 
may as well be the same for each discrete record in the data vector $x_{i=1,2, \ldots, N}$, the discrete calculation will be approximately as shown in Equation (5):

$$
\int_{-\infty}^{+\infty}|x|^{l} p(x) d x=\frac{\sum_{i=1}^{N}\left|x_{i}\right|^{l}}{N}=E\left(|x|^{l}\right)
$$

and (6):

$$
D I_{x}=\frac{\sqrt[l]{E\left(|x|^{l}\right)}}{\sqrt[m]{E\left(|x|^{m}\right)}}
$$

where $\sqrt[l]{E\left(|x|^{l}\right)} \approx \max \left|x_{i}\right|_{i=1,2, \ldots, N}$, if $l \rightarrow+\infty$.

Several dimensionless signal features frequently used are interpreted in DI definition as shown in Table 1. In fact, the first six of them have been extensively utilized for fault diagnosis of rolling bearings, while the last two with their extended forms have been typically applied to battery fault diagnosis.

\begin{tabular}{|c|c|c|}
\hline Signal Feature & DI Interpretation and Calculation Formula & Discrete GDI Interpretation \\
\hline $\begin{array}{c}\text { Waveform indicator } \\
W I_{y}=\frac{\left[\int_{-\infty}^{+\infty},|y|^{2}, p,(y), d, y\right]^{\frac{1}{2}}}{\int_{-\infty}^{+\infty}|y| p(y) d y}\end{array}$ & $\begin{array}{l}\qquad 1=2, m=1 \\
\qquad W I_{y}=\frac{\sqrt{E\left(|y|^{2}\right)}}{E(|y|)} \\
\text { Traditional DI formula [37] }\end{array}$ & 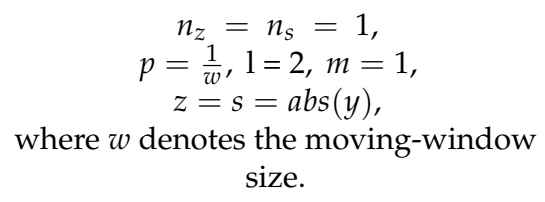 \\
\hline $\begin{array}{l}\text { Impulse indicator } \\
I I_{y}=\frac{\max \left|y_{i}\right|_{i=1,2, \ldots, N}}{\int_{-\infty}^{+\infty}|y| p(y) d y}\end{array}$ & $\begin{array}{c}\qquad \rightarrow \infty, m=1 \\
I I_{y}=\frac{\lim _{l \rightarrow+\infty} \sqrt[l]{E\left(|y|^{l}\right)}}{E(|y|)} \\
\text { Traditional DI formula }\end{array}$ & $\begin{array}{l}n_{z}=n_{s}=1, p=\frac{1}{w} \\
l \rightarrow \infty, m=1 \\
z=s=a b s(y)\end{array}$ \\
\hline $\begin{aligned} \text { Margin indicator } \\
M I_{y}=\frac{\max \left|y_{i}\right|_{i=1,2, \ldots, N}}{\left[\int_{-\infty}^{+\infty}|y|^{\frac{1}{2}} p(y) d y\right]^{2}}\end{aligned}$ & $\begin{array}{c}\qquad l \rightarrow \infty, m=\frac{1}{2} \\
M I_{y}=\frac{\lim _{l \rightarrow+\infty} \sqrt[l]{E\left(|y|^{l}\right)}}{[E(\sqrt{|y|})]^{2}} \\
\text { Traditional DI formula }\end{array}$ & $\begin{array}{c}n_{z}=n_{s}=1, p=\frac{1}{w} \\
l \rightarrow \infty, m=\frac{1}{2} \\
z=s=\operatorname{abs}(y)\end{array}$ \\
\hline $\begin{array}{c}\text { Peak indicator } \\
P I_{y}=\frac{\max \left|y_{i}\right|_{i=1,2, \ldots, N}}{\left[\int_{-\infty}^{+\infty}|y|^{2} p(y) d y\right]^{\frac{1}{2}}}\end{array}$ & $\begin{array}{c}\qquad \rightarrow \infty, m=2 \\
P I_{y}=\frac{\lim _{l \rightarrow+\infty} \sqrt[l]{E\left(|y|^{l}\right)}}{\sqrt{E\left(|y|^{2}\right)}} \\
\text { Traditional DI formula }\end{array}$ & $\begin{array}{l}n_{z}=n_{s}=1, p=\frac{1}{w} \\
l \rightarrow \infty, m=2 \\
z=s=a b s(y)\end{array}$ \\
\hline $\begin{array}{l}\text { Skewness indicator } \\
S I_{y}=\frac{\int_{-\infty}^{+\infty}|y|^{3} p(y) d y}{\sigma^{3}}\end{array}$ & $\begin{array}{c}\qquad \varepsilon=3, \eta=2 \\
S I_{y}=\frac{E\left(|y|^{3}\right)}{E\left(|y|^{2}\right)^{\frac{3}{2}}} \\
\text { Redefined DI formula [38] }\end{array}$ & $\begin{array}{c}n_{z}=n_{s}=3, p=\frac{1}{w} \\
l=3, m=2 \\
z_{1,2,3}=s_{1,2,3}=a b s(y)\end{array}$ \\
\hline $\begin{array}{l}\text { Kurtosis indicator } \\
K I_{y}=\frac{\int_{-\infty}^{+\infty}|y|^{4} p(y) d y}{\sigma^{4}}\end{array}$ & $\begin{array}{c}\varepsilon=4, \eta=2 \\
K I_{y}=\frac{E\left(|y|^{4}\right)}{E\left(|y|^{2}\right)^{2}} \\
\text { Redefined DI formula }\end{array}$ & $\begin{array}{c}n_{z}=n_{s}=4, p=\frac{1}{w} \\
l=4, m=2 \\
z_{1,2,3,4}=s_{1,2,3,4}=\operatorname{abs}(y)\end{array}$ \\
\hline $\begin{array}{c}\text { Entropy indicator } \\
E I_{y}=-\int_{-\infty}^{+\infty} \ln y p(y) d y \\
\text { where } y_{i} \text { denotes the normalized value. }\end{array}$ & DI formula invalid so far & $\begin{array}{c}n_{z}=n_{s}=1, p\left(y_{i}\right)=y_{i} \\
\quad l=m=1 \\
z=-\ln (y), s=\{1\}_{N \times 1}\end{array}$ \\
\hline
\end{tabular}

Table 1. Dimensionless Signal Features [30] and Corresponding Interpretations.

Pearson correlation indicator

$C I_{x_{1}, x_{2}}=\frac{\operatorname{cov}_{x_{1}, x_{2}}}{\sigma_{x_{1}} \sigma_{x_{2}}}=$

$\sum_{i=1}^{N}\left(x_{1, i}-E x_{1}\right)\left(x_{2, i}-E x_{2}\right)$

$\frac{\sum_{i=1}^{N}\left(x_{1, i}-E x_{1}\right)\left(x_{2, i}-E x_{2}\right)}{\sqrt{\sum_{i=1}^{N}\left(x_{1, i}-E x_{1}\right)^{2}} \sqrt{\sum_{i=1}^{N}\left(x_{2, i}-E x_{2}\right)^{2}}}$

DI formula invalid so far

Composed of 2 sub-GDIs, see Appendix A in detail

where $E x=\int_{-\infty}^{+\infty} x p(x) d x$. 
It can be inferred from Table 1 that the first four features strictly follow the DI construction formula in Equation (4), but are hardly the same as the others, particularly in the last two cases. That is, though correlation coefficient and entropy have been widely used in signal-based battery fault diagnosis study, there is a lack of generalized formula to realize a comprehensive coverage along with the others efficiently. Moreover, redefined dimensionless indicators (RDIs) under separately discussed conditions [38] are as shown in Equation (7):

$$
\left\{\begin{array}{c}
\zeta_{1}=\frac{\left[\int_{-\infty}^{+\infty}|y|^{\alpha} p(y) d y\right]^{\frac{1}{\alpha}}}{\left[\int_{-\infty}^{+\infty}|y|^{\beta} p(y) d y\right]^{\frac{1}{\beta}}}=\frac{\sqrt[\alpha]{E\left(|y|^{\alpha}\right)}}{\sqrt[\beta]{E\left(|y|^{\beta}\right)}} \\
\zeta_{2}=\lim _{l \rightarrow+\infty} \frac{\left[\int_{-\infty}^{+\infty}|y|^{l} p(y) d y\right]^{\frac{1}{l}}}{\left[\int_{-\infty}^{+\infty}|y|^{\gamma} p(y) d y\right]^{\frac{1}{\gamma}}}=\lim _{l \rightarrow+\infty} \frac{\sqrt[l]{E\left(|y|^{l}\right)}}{\sqrt[\gamma]{E\left(|y|^{\gamma}\right)}} \\
\zeta_{3}=\frac{\int_{-\infty}^{+\infty}|y|^{\varepsilon} p(y) d y}{\left[\int_{-\infty}^{+\infty}|y|^{\eta} p(y) d y\right]^{\frac{1}{\eta}}}=\frac{E\left(|y|^{\varepsilon}\right)}{\left[E\left(|y|^{\eta}\right)\right]^{\frac{\varepsilon}{\eta}}}
\end{array}\right.
$$

where constants $\alpha, \beta, \gamma \in[0.5,5]$ are assigned with a step size of $\lambda=0.5$, and constant $\varepsilon \in[1,5], \eta \in[0.5,5](\varepsilon \neq \eta)$ are respectively assigned with step size $\lambda_{1}=1, \lambda_{2}=0.5$. In this way, hundreds of different RDIs could be obtained and well-chosen with various selection methods, but it is obvious that there is a lack of relevant physical meaning to explicitly account for every one of them, thus impeding subsequent fault identification. Besides, by means of introducing additional variables to imply different signal sources for the numerator and the denominator, the machinery trouble-free signals and the characteristics of the fault signals were together included in the DI definition [39]. Based on these studies, the GDIs are hereby defined as Equation (8):

$$
G D I=\frac{\prod_{i=1}^{n_{z}}\left[\int_{-\infty}^{+\infty} \mathrm{z}_{i}^{l_{i}} p_{i}\left(\mathrm{z}_{i}\right) d z_{i}\right]^{\frac{1}{l_{i}}}}{\prod_{j=1}^{n_{s}}\left[\int_{-\infty}^{+\infty} \mathrm{s}_{j}^{m_{j}} p_{j}\left(\mathrm{~s}_{j}\right) d s_{j}\right]^{\frac{1}{m_{j}}}}
$$

where $z_{i}, s_{j}$ denote the simply processed signal vectors from single or multiple signal sources or constants, $\mathrm{p}(\cdot)$ denotes the PDF of signal records, $\mathrm{l}_{\mathrm{i}}, \mathrm{m}_{\mathrm{j}}$ are positive constants, and $n_{z}, n_{s}$ denote the order of GDIs in terms of signal source complexity, as many features are first-order indexes as the first half in Table 1. The steps of the feature construction and extraction part are as follows:

- Firstly, the collected voltage data records should be divided into segments. The components of each segment are obtained after VMD analysis. To reveal cell inconsistency, the static parts will be efficiently used.

- Then, the decomposed components of each segment, especially the dynamic parts, are observed in moving windows with a proper size determined by the actual sampling frequency. In order to enhance the flexibility of the formula, $z_{i}, s_{j}$ are obtained after processing the decomposed components, like data standardization and scale transformation with simple functions.

- Next, constant $l_{i}, m_{j}$ are assigned positive integers, for $l_{i}, m_{j}$-order roots of the integrals $\left[\int_{-\infty}^{+\infty} z_{i}^{l_{i}} p_{i}\left(z_{i}\right) d z_{i}\right]^{\frac{1}{l_{i}}}$ and $\left[\int_{-\infty}^{+\infty} s_{j}^{m_{j}} p_{j}\left(s_{j}\right) d s_{j}\right]^{\frac{1}{m_{j}}}$ can be theoretically regarded as the first moment, second moment, third moment etc. of $z_{i}, s_{j}$.

- Finally, the order of a GDI is evaluated by the supplemental constant $n$, which means that there may be not more than $n$ potential signal sources with different signal dimensions, as seen in the correlation indicator, or $n$ th-power of $l_{i}, m_{j}$ th-moment of a data vector from a certain signal resource, such as the skewness indicator and the kurtosis indicator, or combination of these scenarios. 
It is noteworthy that, there could be different values of $n_{z}$ and $n_{s}$, as the correlation indicator interpretation further proved in the Appendix A. In contrast to those existing DI extraction methods, the construction procedure of GDI is accompanied with additional physical significance instead of value assignment with a certain interval in reasonable ranges to parameters in the formula and then seeking for the ones containing distinct discrepancy among them, which is critical for further identification of the abnormal signal pattern. This can be realized by the following fact that, the positivity and negativity of the identified outliers in different kinds of feature sequences can also be considered as a useful indicator of specific abnormal patterns in the moving-window observed voltage signals, such as overvoltage/undervoltage, and abnormal rising/falling tendencies.

Note that in order to eliminate the end effect and mode mixing, both $\mathrm{z}$ and $\mathrm{s}$ equal signed voltage signals minus the average sequence of static components. As a typical set of the preferable parameters, the discrete parameter values in this paper are assigned $n=3, l=3, m=2, p=1 / w$ shown in Figure 3 for the following illustrations.

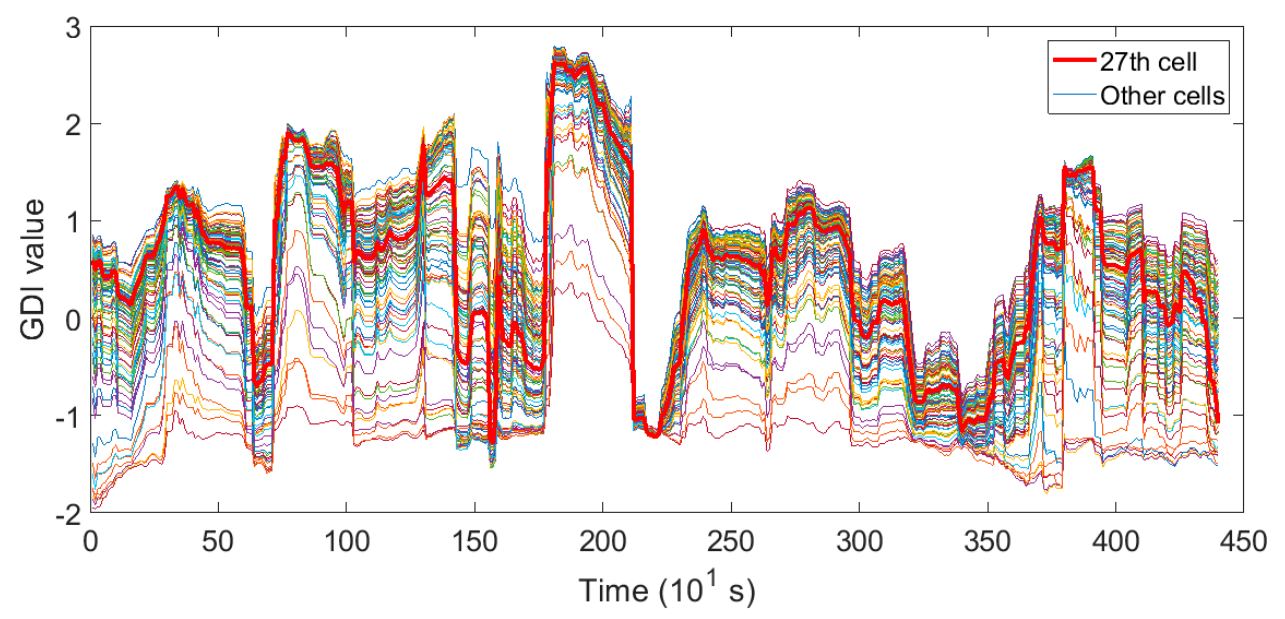

Figure 3. GDI waveforms in the first case.

\section{Clustering upon Feature Sequences}

As a prevailing unsupervised learning method, clustering is adopted in this paper to find the outliers that represent cell abnormalities in feature sequences. Generally, there are three kinds of ideas for clustering [40]:

- Normal data records belong to a cluster in the data, whereas anomalies don't belong to any cluster;

- Normal data records lie close to their closest cluster centroid, whereas anomalies are far away from their closest cluster centroid;

- Normal data records belong to large and dense clusters, while anomalies either belong to small or sparse clusters.

For the first kind of clustering methods, they are not optimized to find battery cells with the abnormalities and the inducements directly, such as density-based spatial clustering of applications with noise (DBSCAN) clustering and spiking neural networks (SNN), because the main aim of the underlying clustering algorithm in this paper is to obtain the clusters from the feature sequences without completely known thresholds in advance. Besides, a certain outlier may become the centroid of one cluster itself, and the techniques satisfying the second will be invalid to detect such anomalies, such as $k$-means clustering and self-organizing maps (SOMs). Therefore, utilizing clustering algorithms conforming to the third is preferable in this paper, which means that, the specific cell data records would be abnormal if the density of their clusters were lower than a threshold. In essence, it is more feasible and convincible to determine the actual quantity of abnormal cells (usually starting from one single cell in reality) that may lead to a devastating consequence, than to determine a certain adaptive-questionable threshold by supervised learning with very 
limited training data. In fact, after implementing the clustering algorithm optimized by searching for the least sum of centroid distances, clusters that contain only very few records in feature sequences indicate potential cell abnormalities.

Here, two types of sequences related to GDIs are utilized as the main material for anomaly detection in the proposed method: the deviation from the mean GDI sequence, and the forward difference of the deviation sequences shown in Figure 4. Certainly it is available to observe the variation tendency of the sequences with longer time scales, but the typical range of voltage sampling frequency is $10^{-1}$ to $10^{0} \mathrm{~Hz}$, we pay attention to the horizontally-compared difference for detailed investigation, along with the difference of the GDI sequences within a relatively short period. That is to say, the difference in the variation of original signals between the last moments of temporal/previous observation, can be assessed in many dimensionless ways, so that abnormal patterns could be detected sensitively and intuitively by finding out the outliers in various sequences made of GDIs.
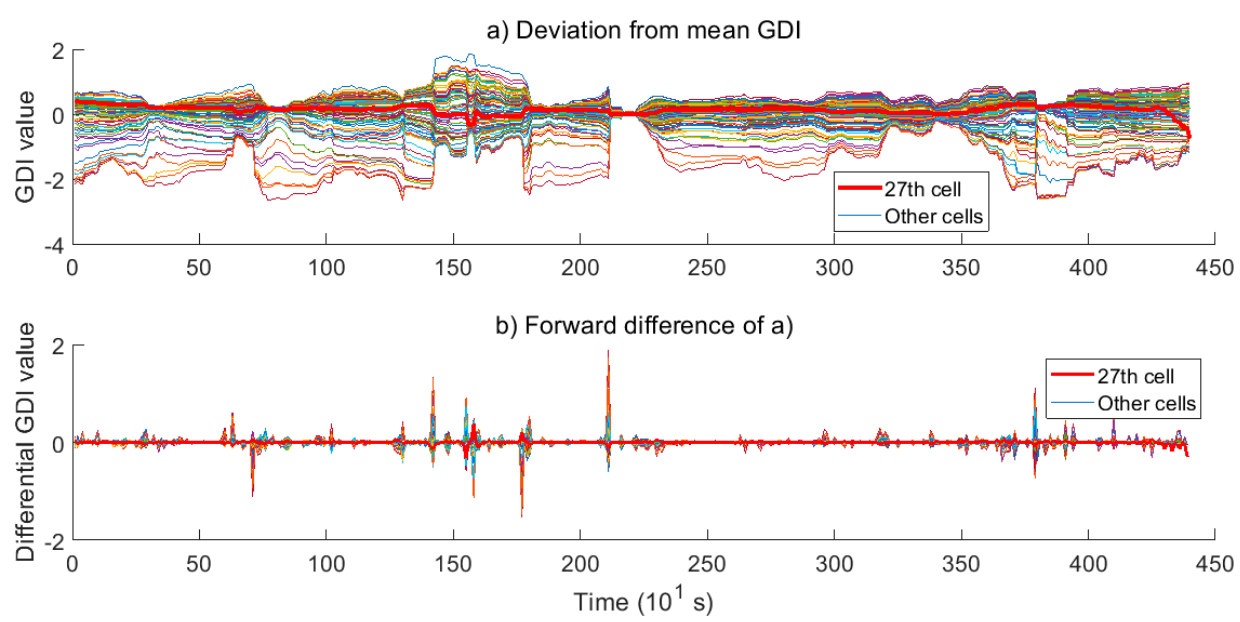

Figure 4. Extracted GDI sequences in the first case, (a) the deviation from the mean GDI sequence; (b) forward difference of (a).

To make quantified evaluations, time-priority is one of the most important factors to consider, by which the degree of cell abnormality can be determined and accumulated. If the sparsity degree of a certain cluster with minimum GDI units at moment $t$ is low enough, the damping factor $e^{-d}$ is firstly introduced in the procedure of anomaly evaluation to manifest the impact of time-priority in a specific segment. Then in order to make horizontal comparisons, absolute values of the minor ones divided by the standard deviation at corresponding moments are added to the anomaly counting vector as shown in Equation (9):

$$
C_{\text {abnor }}(t)=\left\{\begin{array}{c}
\frac{\left|G D I_{k}(t)\right|}{\operatorname{std}(G D I(t))}, t=1 \\
e^{-d} \cdot C_{a b n o r}(t-1)+\frac{\left|G D I_{k}(t)\right|}{\operatorname{std}(G D I(t))}, t \geq 2
\end{array}\right.
$$

where $C_{a b n o r}(t)$ denotes the vector to accumulate the degree of abnormality in current data segment up to moment $t, G D I_{k}(t)$ denotes the feature vector including detected sparse clusters whose member amount is less than threshold $k$ (preferably 2 ) at sample moment $t$, $d$ denotes the non-negative exponential damping constant.

If it is required to investigate some very temporal details in online applications, constant $d$ is assigned a large positive value, so that the horizontal comparison shall play a leading role in the evaluation procedure. In contrast, the analysis on multiple integrated historical voltage data segments may prefer a small positive value for constant $d$, to achieve an overall judgment on contributions at different detected moments of similar weights. As a consequence, the quantified degree of cell abnormality can be determined moment by moment as shown in Figure 5, with very little contributions to $C_{a b n o r}$ from actually 
normal cells at potentially misclassified valid moments. In this way, false alarms can be significantly reduced, compared with classical threshold-based supervised learning methods, such as $3 \sigma$ principle thresholds, and quantile linear thresholds. Finally, the sequence of $C_{a b n o r}$ can be obtained by applying the proposed algorithm to a few real-time voltage data vectors, or even a flexible amount of historical pack voltage data segments adaptively, verified in the following section.

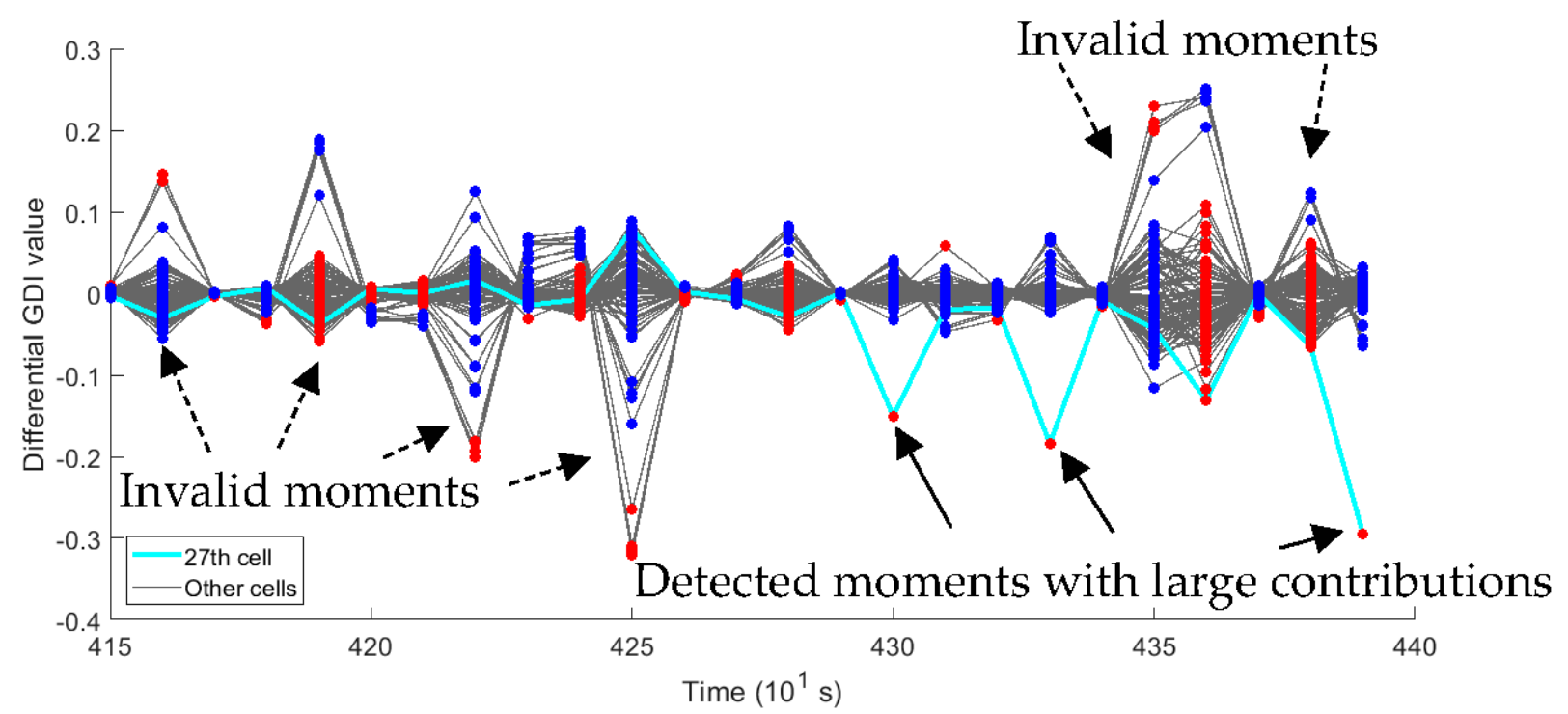

Figure 5. Zoom-in scatter plots of the sparsity-based clustering in the first case, with the points in different temporal clusters marked in red or blue.

\section{Results and Discussion}

Comparisons of the results obtained by implementing different techniques from the existing studies are made in this section. There are three practical fault cases of electric vehicles driven by batteries with $\mathrm{Li}(\mathrm{NiMnCo}) \mathrm{O}_{2}$ cathodes, and the results will be respectively discussed below. The voltage sampling frequency of all cases is $0.1 \mathrm{~Hz}$ with $1 \mathrm{mV}$ resolution. The detailed algorithms chosen to make comparisons with our proposed method are the correlation-based method, the entropy-based method with data normalization, and the threshold-based method by the practical BMS. However, considering that there may be either a fixed threshold or a time-varying threshold to determine, eliminating as many false alarms as possible is an important objective, with the same size of the moving window in each case to observe the voltage signals.

\subsection{Case 1: Pre-Fault Data up to BMS Alarm of a Thermal Runaway Accident}

The pre-fault dataset starts from the last discharging segment and ends at the 440th moment of credible BMS detection as shown in Figure 2. It can be inferred from Figure 5 that outliers are seen at the 430th, the 433rd, and the 439th moment, and negative values of the differential GDIs can indicate an abnormal falling tendency of the 27th cell's voltage. In turn, positive values of the detected outliers could imply that abnormal rising of cell voltage data takes place.

Using a fixed threshold, such as 0.5 typically used in [27], no credible detection can be made by correlation coefficients in Figure 6a. Besides, the quantity of false detections will dramatically increase if a time-varying threshold is adaptively used to investigate the whole segment. After using a fixed threshold, the earliest credible detection could be given at the 433rd moment by the correlation-based method, but the specific voltage data performance of the abnormal cell remains unknown, because correlation coefficients can be significantly reduced by diversified means. 

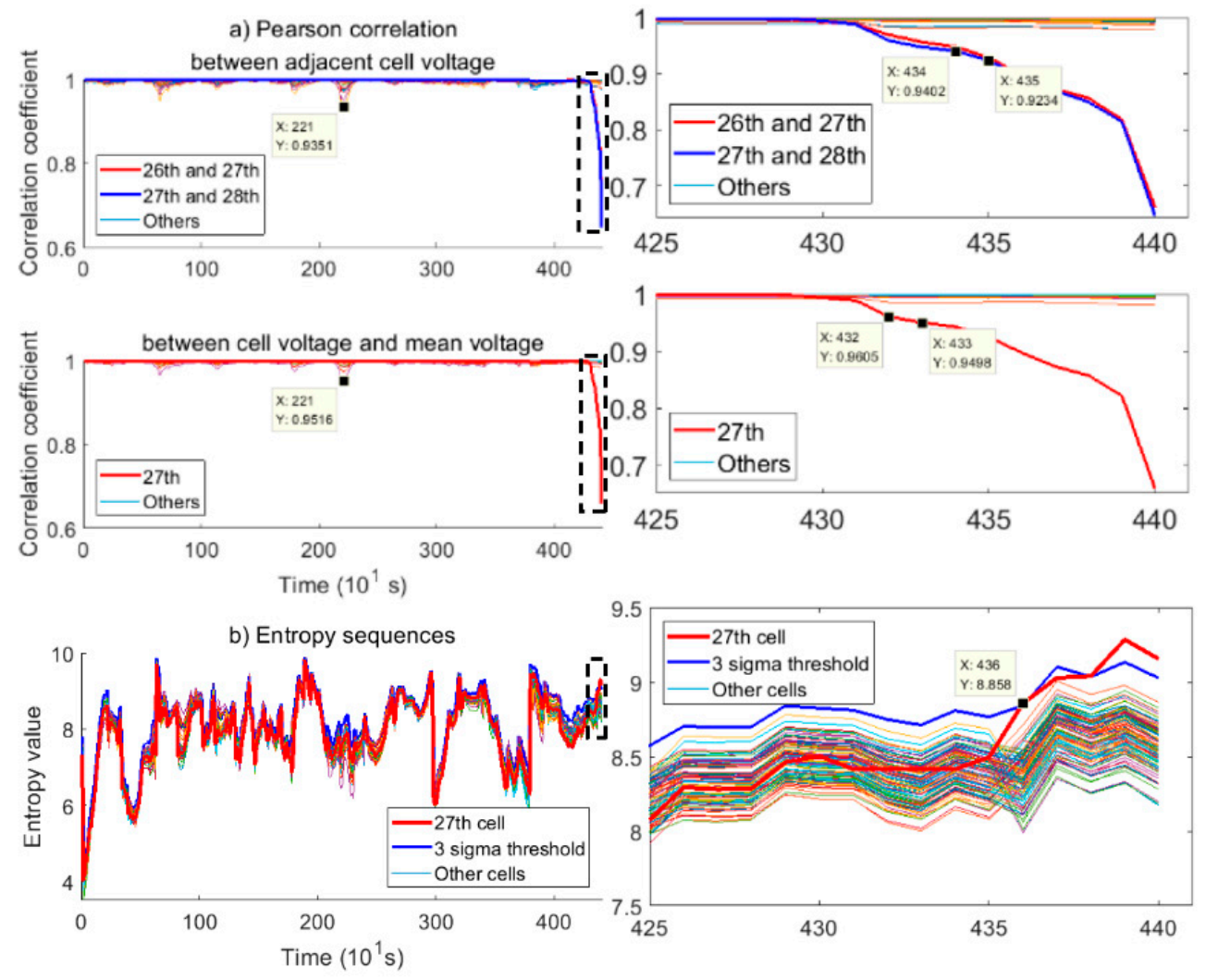

Figure 6. From the pre-fault dataset in the first case, (a) left: Sequences of Pearson correlation coefficients; right: Zoom-in of the parts bounded by dotted lines (b) left: Sequences of the entropy of normalized cell voltage data; right: Zoom-in of the part bounded by dotted lines.

As shown in Figure $6 \mathrm{~b}$, the earliest outlier can be logically detected with $3 \sigma$ thresholds at the 436th moment, but the quantity of false detections is extremely disappointing.

The results are shown in Table 2. Since no precursory signals are credible before the voltage plunging, the earliest fault detection confirms the excellence of the sensitivity of the proposed method.

Table 2. Performance of the Methods in Case 1.

\begin{tabular}{cccc}
\hline Method & First Credible Detection & Fault Identification & False Detection \\
\hline Differential voltage threshold by BMS & 440 th & Voltage drop & None \\
\hline Correlation coefficient & $433 \mathrm{rd}$ & Incapable & None \\
\hline Entropy & $436 \mathrm{th}$ & Incapable & Lots of uncertainties \\
\hline Proposed GDI clustering & 430 th & Abnormal undervoltage & Little contribution to $C_{a b n o r}$ \\
\hline
\end{tabular}

5.2. Case 2: Voltage Data up to the First BMS Alarm (the 1280th Moment) with Intermittent Inconspicuous Anomaly Occurrence

In the second case, frequent occurrence of inconspicuous under-voltage can be found in the historical voltage signals, and BMS detection was triggered at the 1280th moment. The dataset as shown in Figure 7 begins from the beginning of the uploaded data, containing multiple charging and discharging segments. Four relevant undervoltage instances are mainly concerned in the case shown in subplots of Figure 7. 

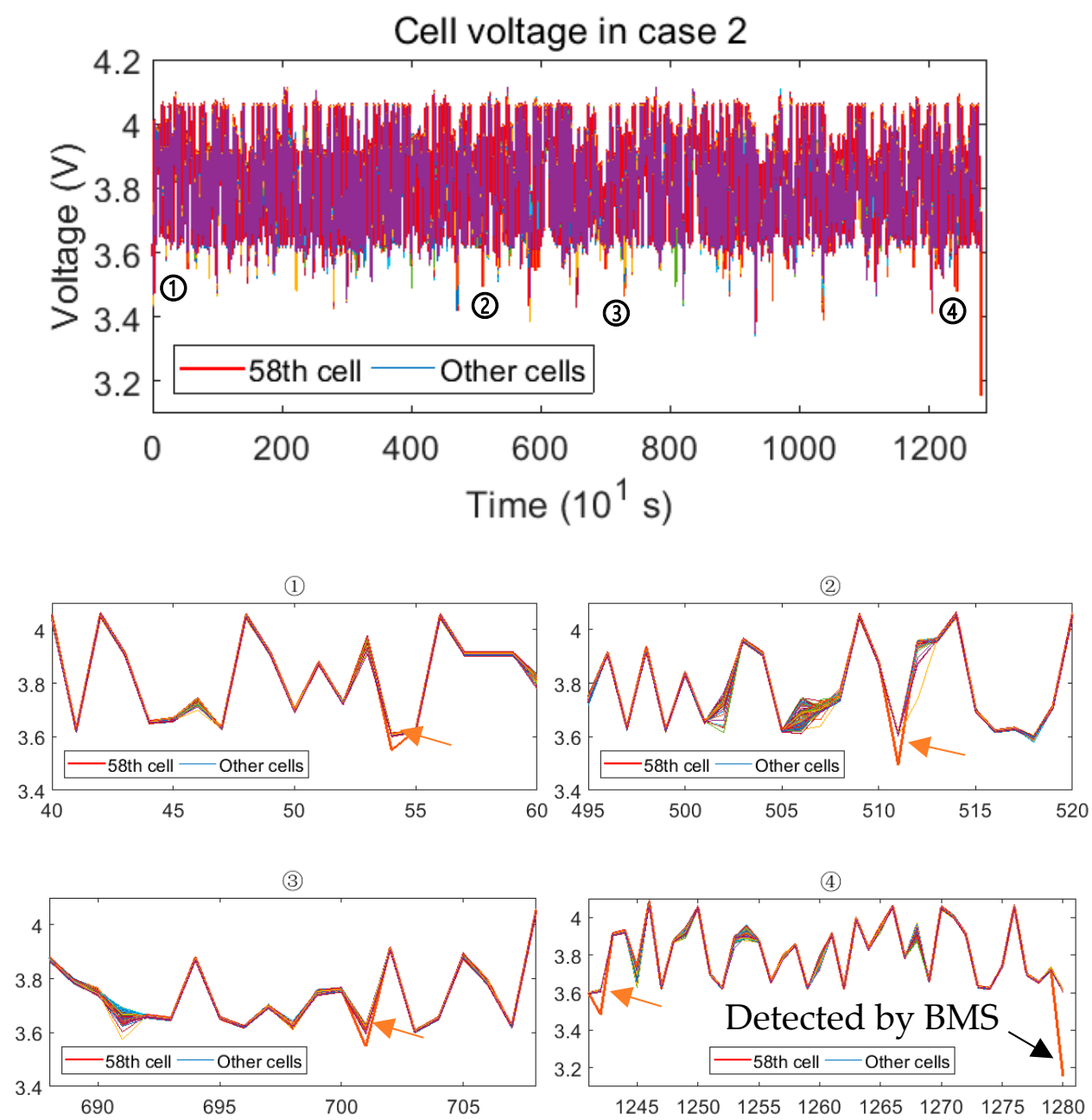

Figure 7. Voltage signal records of 90 battery cells in the second case; subplots: zoom-in of 4 concerned parts containing under-voltages (with orange arrows).

Before the final moment detected by BMS, anomaly detection cannot be realized by correlation coefficients without false detections, regardless of whether a time-varying threshold or a fixed threshold is used, as shown in Figure 8a.

As for using an entropy-based detection as shown in Figure $8 b$, it is appropriate to adopt a time-varying threshold compared with a fixed one, then the anomaly detections can be made roughly in accordance with the proposed method as in Figure 8c. However, it is still incapable of acquiring the knowledge about the specific voltage data performances of the abnormal cell, and false alarms take place frequently in the entropy sequences.

It can be inferred from the result of the proposed method that, after using the proposed method, four successful outlier detections can be credibly made in the concerned local parts. Then we make a little discussion on the abnormal performance identification in this typical case below.

The abnormal pattern of voltage signals of the relevant cell can be recognized as multiple short-time under-voltage instances of the 58th cell, because positive outliers in differential GDI deviation sequence of that cell can be observed exactly a window size later from negative outliers of the same cell. In fact, the duration of a short voltage pulse could be determined by subtracting the moving window size from the time interval of the proposed feature's sign reversion. In turn, if an overvoltage pulse emerges, the sign of the outliers in the sequences will change from positive when the pulse slides into the moving window observed, to negative as the pulse slides out. 
a) Pearson correlation
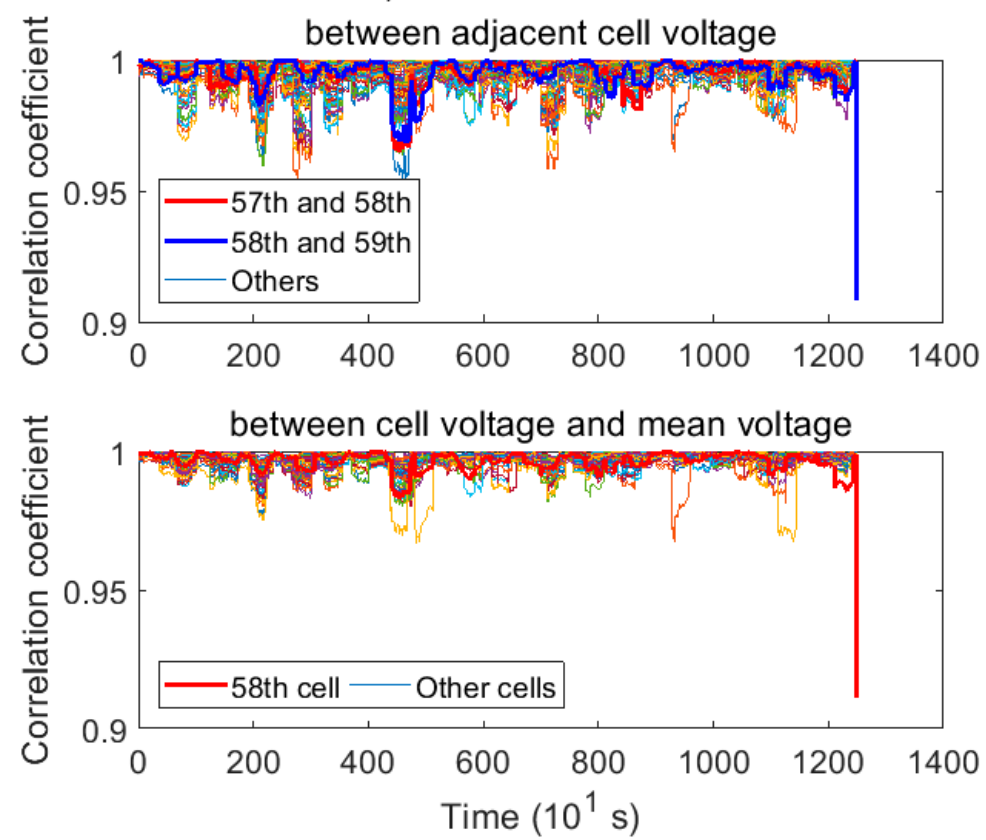

b) Entropy sequences
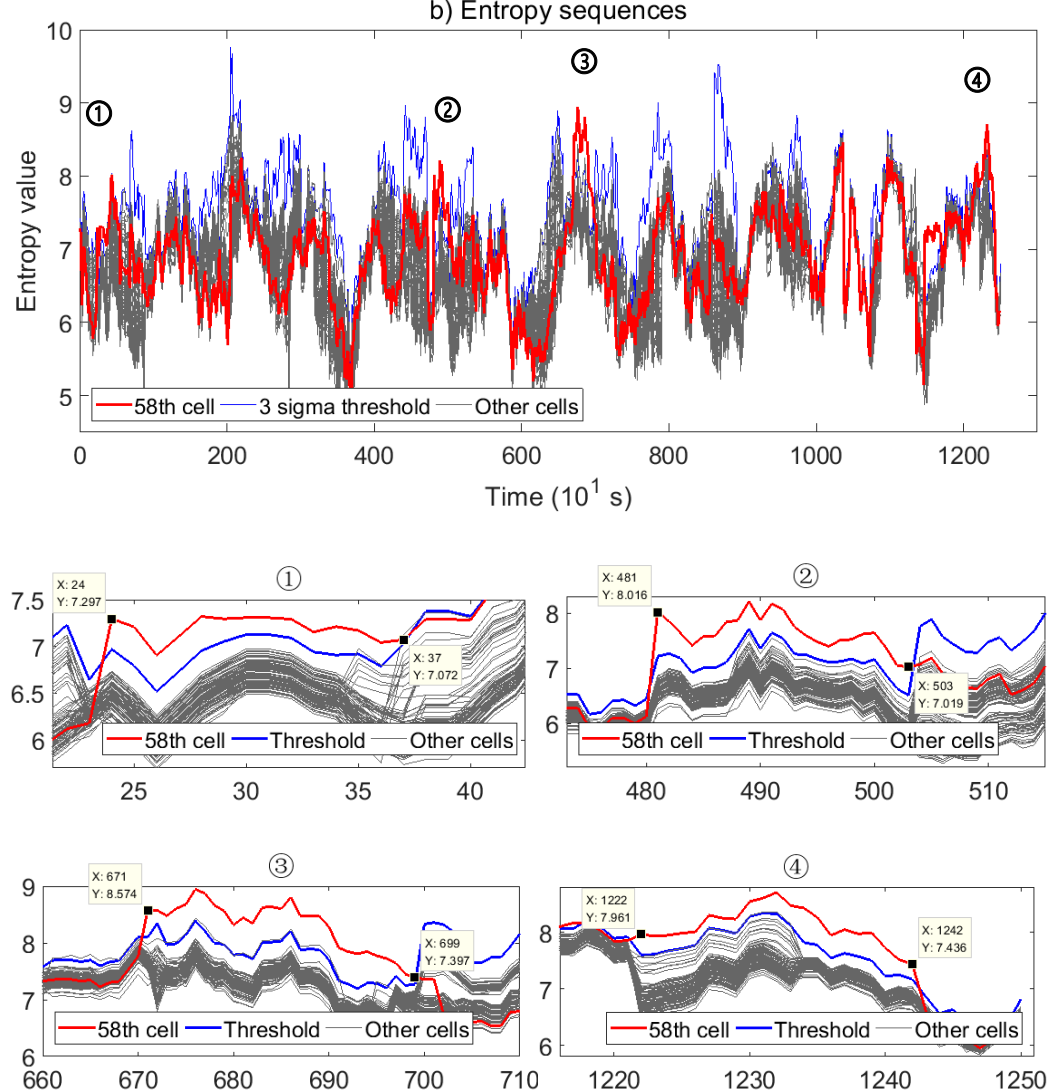

Figure 8. Cont. 

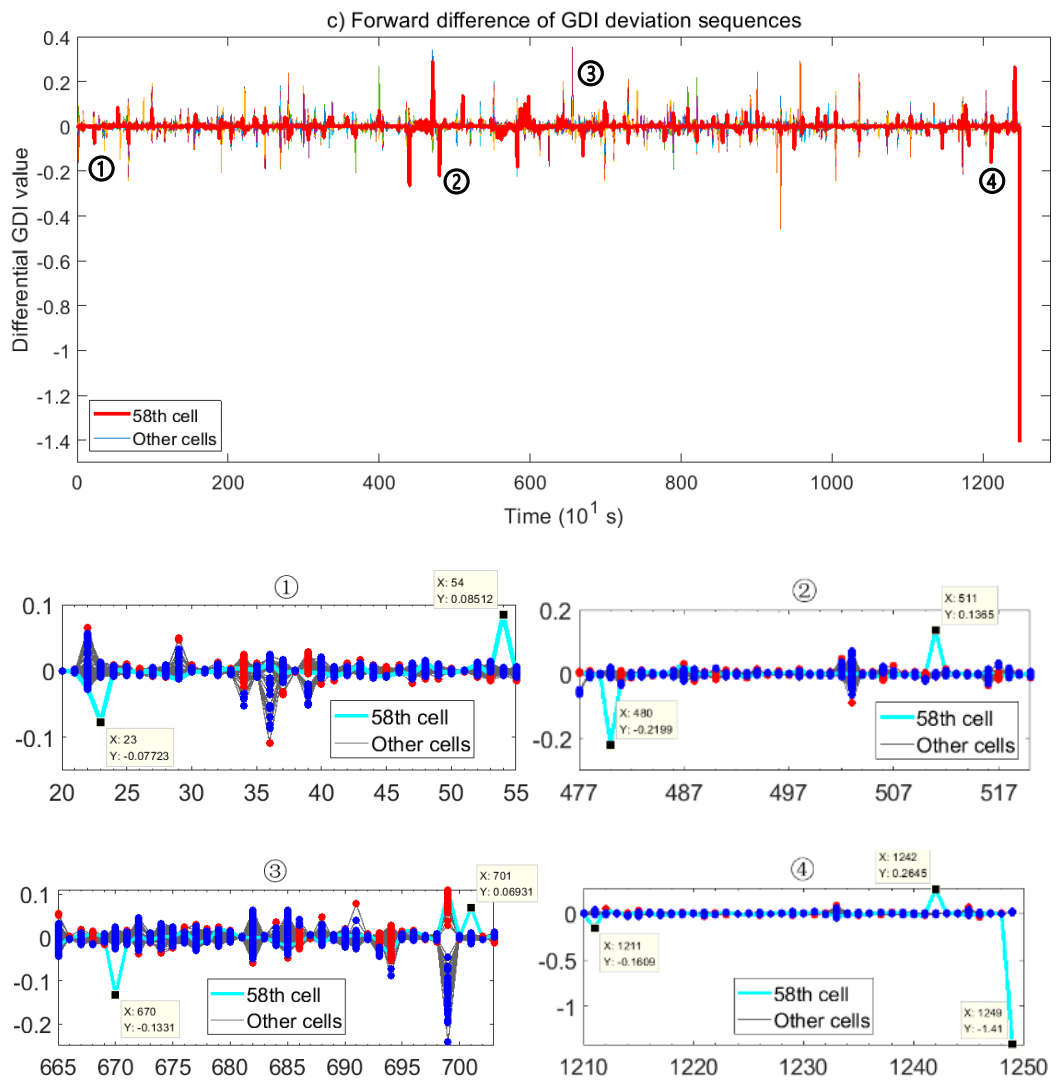

Figure 8. From the pre-fault dataset in the second case, (a) Sequences of Pearson correlation coefficients; (b) Sequences of the entropy of normalized cell voltage data; subplots: Zoom-in of 4 concerned parts; (c) Forward difference of the GDI deviation sequences; subplots: Zoom-in of 4 corresponding under-voltages, with the points in different temporal clusters marked in red or blue.

The results are listed in Table 3, and there is also a lack of definitive overall conclusion by using the entropy-based method, in spite of the similar detections made in the concerned local parts. The comprehensive result in Figure 9 indicates that, despite the short fluctuation at the beginning, the 58th cell has been evaluated as the most abnormal cell, starting from the 472nd moment to the last moment with the damping factor $d=0.1$ in (9) by the proposed method. To sum up, types, durations, and severity of local abnormalities can be effectively identified by the proposed method, together with a comprehensive evaluation that is steadily attained as early as possible, on cell abnormalities over multiple segments in this case.

Table 3. Performance of the Methods in Case 2.

\begin{tabular}{|c|c|c|c|c|}
\hline Method & Diagnosis & Definitive Judgment & $\begin{array}{c}\text { Moments of } \\
\text { Significant Detections } \\
\text { for Concerned Parts }\end{array}$ & False Detection \\
\hline $\begin{array}{l}\text { Differential voltage } \\
\text { threshold by BMS }\end{array}$ & Voltage drop detected & 1280th only & $\begin{array}{l}\text { Obvious drop at the } \\
\text { 1280th moment only }\end{array}$ & None \\
\hline Correlation coefficient & Failed to detect & - & - & - \\
\hline Entropy & $\begin{array}{l}\text { Detected, incapable of } \\
\text { identification }\end{array}$ & Uncertain & $\begin{array}{l}24 \text { th to } 37 \text { th, } 481 \text { st to } \\
503 \text { rd, } 671 \text { st to } 699 \text { th, } \\
\text { and } 1222 \text { nd to } 1242 \text { nd }\end{array}$ & Lots of \\
\hline $\begin{array}{l}\text { Proposed GDI } \\
\text { clustering }\end{array}$ & $\begin{array}{l}4 \text { undervoltages } \\
\text { identified }\end{array}$ & Since 472 nd by $C_{a b n o r}$ & $\begin{array}{l}\text { 23rd \& 54th, 480th \& } \\
\text { 511st, 670th \& 701st, } \\
\text { and 1211st \& 1242nd }\end{array}$ & $\begin{array}{l}\text { Little contribution to } \\
\qquad C_{a b n o r}\end{array}$ \\
\hline
\end{tabular}




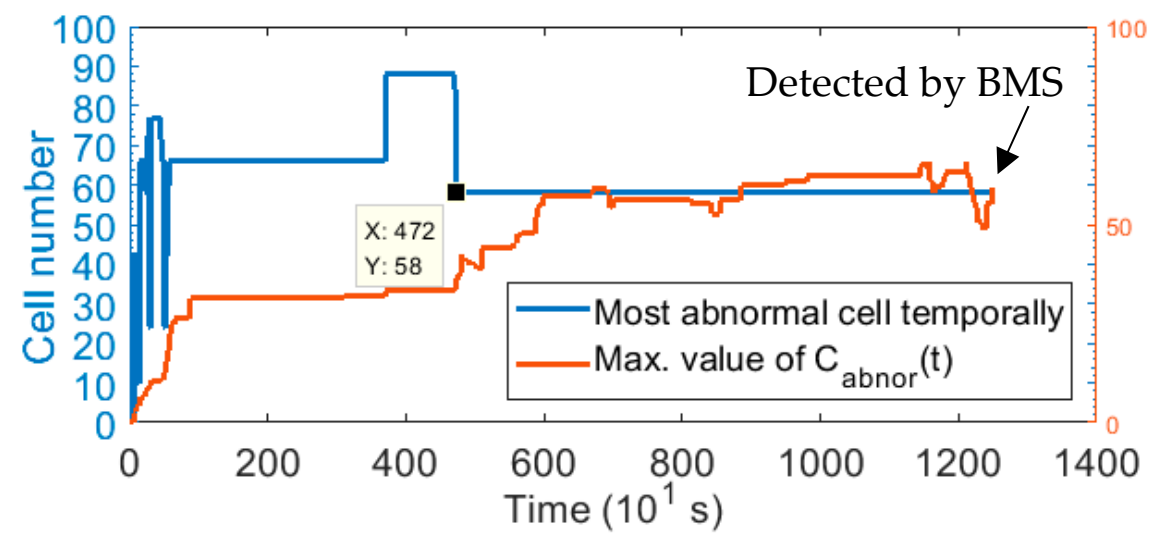

Figure 9. The most abnormal battery cell temporally, determined by the cell with the largest $C_{a b n o r}(t)$ with $d=0.1$ in case 2 .

\subsection{Case 3: Last Discharging Segment Before Fault Occurrence during Fast Charging}

In the third case, due to historical data accessibility of the electric vehicle that suffered a very severe fault in a fast charging process, the utilized dataset shown in Figure 10 is the whole last discharging segment before the fault occurrence when it was charged.

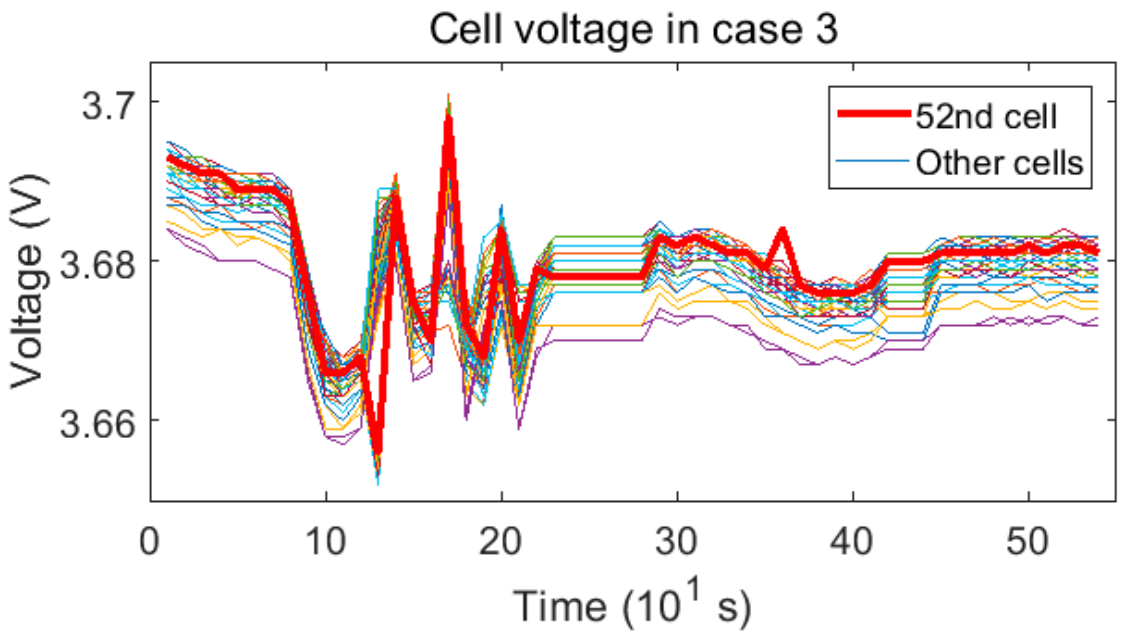

Figure 10. Voltage signal records of 90 battery cells in the third case.

The dataset shown in Figure 10 contains two different types of patterns that are recorded in actual vehicle operation: aggresively varying discharging with excessively long sampling intervals, thus failed to immediately trace the accurate transient variation of voltage signals in the first half, and a steady voltage reduction with a little standby-mode fluctuation in the second half. By virtue of easily implemented manual temporal-series examinations upon the short dataset, an overvoltage pulse of the 52nd cell can be considered to be the major abnormal performance in the second half. Since no anomaly detection by BMS is available as a comparison, the robustness of the methods will be mainly verified in this case.

Adopting a time-varying threshold in the sequence of correlation coefficients, the voltage records of the 52nd cell are considered as outliers from the 24th to 30th moment, but it is likely to make lots of false detections shown in Figure 11a with no further performance interpretation available. Unfortunately, no valid detections related to the 52nd cell could be significantly made by entropy-based methods in Figure $11 \mathrm{~b}$ regardless of the threshold type selected. 
a) Pearson correlation
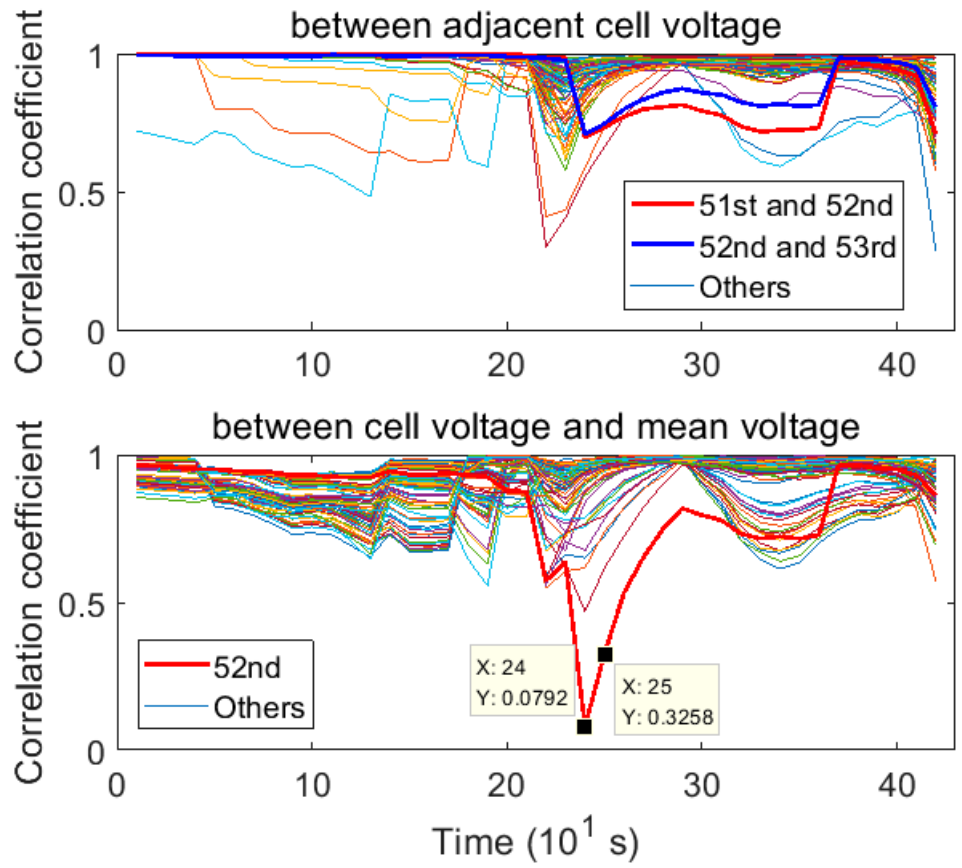

b) Entropy sequences

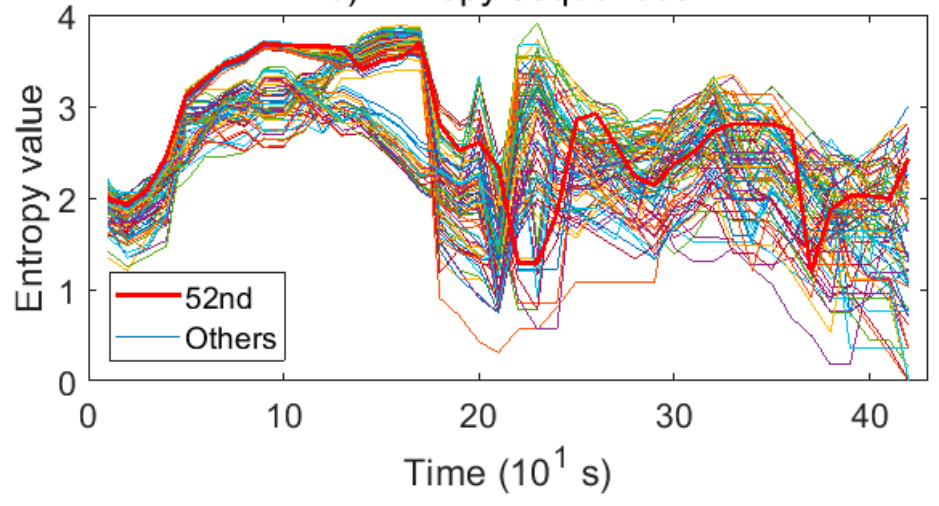

c) Forward difference of GDI deviation sequences

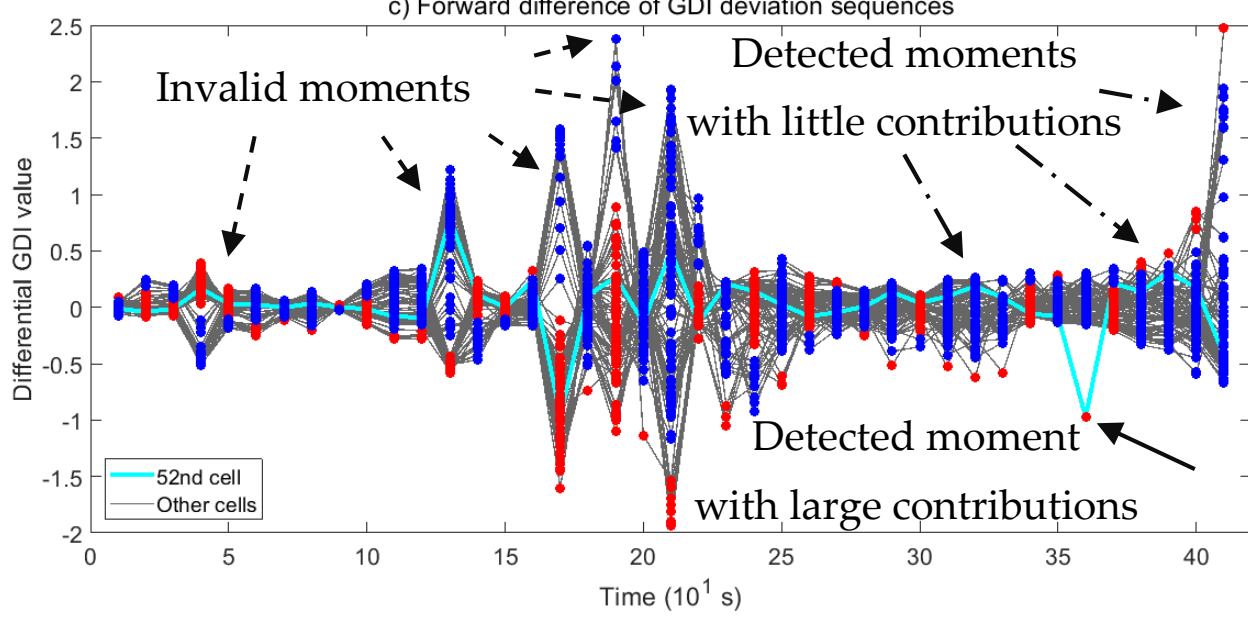

Figure 11. From the pre-fault dataset in the third case, (a) Sequences of the Pearson correlation coefficient; (b) Sequences of the entropy of normalized cell voltage data; (c) The forward difference of the GDI deviation sequences, with the points in different temporal clusters marked in red or blue. 
It can be inferred from the result of the proposed method in Figure 11c that, the moments while aggresively varying voltage amplitudes can be effectively treated as invalid clustering moments in the first half, because the quantity of the feature points in the minor clusters is not low enough, thus avoiding such false alarms. In the second half, an outlier with large negative amplitude at the 36th moment can be recognized and assigned with a large weight value. The $3 \mathrm{mV}$ rising edge of the abnormal voltage pulse is not as obviously detected as its $5 \mathrm{mV}$ falling edge, which makes a positive outlier that should have been obviously detected hidden in the forward difference of the GDI deviation sequences.

The results are listed in Table 4 and provide us with the verification on the balanced performance of sensitivity and stability of the proposed method, thus robust to diversified actual operating conditions.

Table 4. Performance of the Methods in Case 3.

\begin{tabular}{cccc}
\hline Method & Fault Diagnosis & $\begin{array}{c}\text { Moments of } \\
\text { Significant Detections }\end{array}$ & False Detection \\
\hline Differential voltage threshold by BMS & Failed to detect & - & Lots of \\
\hline Correlation coefficient & $\begin{array}{c}\text { Detected, incapable } \\
\text { of identification }\end{array}$ & 24th to 30th & - \\
\hline Entropy & Failed to detect & - & Little contribution to $C_{a b n o r}$ \\
\hline Proposed GDI clustering & Falling detected
\end{tabular}

\section{Conclusions}

In this paper, a comprehensive signal-based fault diagnosis method for lithium-ion batteries in electric vehicles is proposed. The reduced hardware cost attributed to no effort required in battery modeling makes it suitable for online applications, and it is proven that the method can detect the initial stages of the fault, by capturing the abnormal varying tendencies or short-time pulses, with corresponding variation reflected in recognized outliers in various feature sequences. Besides, this method contains the following highlights:

The cell inconsistency in battery states can be separately analyzed by VMD, which is explained from the perspective of different frequency bands of lithium-ion battery responses. The characteristics of dynamic responses under both charging and discharging conditions can get involved in fault diagnosis, whereas the static components can be used in the path of battery aging mechanism investigation and cell state estimation.

Next, the formula of GDI construction is proposed in (8) as the part of signal feature extraction, and existing features in battery fault diagnosis papers, such as correlation coefficient, entropy, and diversified signal indexes have been proven to be particular cases of the generalized formula. Preferable parameter values with a practical significance have been suggested and taken as the example to extract the critical characteristic sequences carried with signed indicators from voltage data signals. The moving window observation with an appropriate size is indispensably adopted here to satisfy the demand on balancing the stability and sensitivity of the method, no matter online or offline. The first-order forward difference of the GDI deviation sequences is representatively used to illustrate the subsequent process of signal fault diagnosis.

Moreover, the excellence of unsupervised learning is verified in signal anomaly detection, because a well-compatible sparsity-based clustering method is utilized to find out the outliers in feature sequences. Credible detections can be made by the proposed judgment, whereas the judgment leads to either a growing number of false negative fault, or frequent false positive faults in other threshold-based ways. In addition, to draw an overall diagnostic conclusion, a damping factor as the time-prior weight factor with temporal standardization of the absolute value is introduced in the comprehensive scores of abnormal degree quantification as in (9). The counting is validated to be very useful for early stage detections if slight abnormalities of a certain battery cell take place intermittently. 
At last, the fault identification is realized by well-founded discussions on the sign variation, intervals, and time-priority weighed standardized absolute values of the outliers in the sequences, so that local abnormal signal patterns can be correctly identified in terms of types, duration, and severity with good robustness, which is hardly feasible in other existing signal-based methods.

The future work is to make modified evaluations based on multiple feature and method fusion from diversified physical signal sensors [41], and to enhance the adaptive ability of thresholds of various algorithms upon different types of batteries. With the development of data acquisition and storage techniques, an increasing number of decomposed signal components from different types of sensors will be available for further specific studies, so that the sensor failure and cell state inconsistency shall be truly isolated.

Author Contributions: Methodology, X.C.; software, X.C.; validation, X.C., C.Z., W.Z. and L.Z.; resources, C.Z., W.Z., J.J. and Y.J.; data curation, X.C. and Y.J.; writing-original draft preparation, X.C.; writing-review and editing, C.Z., J.J., W.Z., Y.J. and L.Z.; funding acquisition, C.Z., W.Z. and J.J. All authors have read and agreed to the published version of the manuscript.

Funding: This research was funded in part by the National Key Research and Development Program of China under Grant 2018YFB0905304 and the National Natural Science Foundation of China, under Grant 61633015, Grant U1664255, and Grant 51977007.

Institutional Review Board Statement: Not applicable.

Informed Consent Statement: Not applicable.

Data Availability Statement: Data available on request due to restrictions by confidentiality regulations of relevant corporations.

Conflicts of Interest: The authors declare no conflict of interest.

\section{Appendix A}

The proof of discrete $C I$ interpretation by the proposed GDI formula is as follows:

$$
C I_{x_{1}, x_{2}}=\frac{\sum_{i=1}^{N}\left(x_{1, i}-E x_{1}\right)\left(x_{2, i}-E x_{2}\right)}{\sqrt{\sum_{i=1}^{N}\left(x_{1, i}-E x_{1}\right)^{2}} \sqrt{\sum_{i=1}^{N}\left(x_{2, i}-E x_{2}\right)^{2}}}
$$

The commonly used form in (A1) is inappropriate for recursive calculation [27] and interpretation, so an equivalent form is obtained after both the numerator and denominator are divided by $N$ as shown in (A2):

$$
\begin{aligned}
C I_{x_{1}, x_{2}}= & \frac{\frac{1}{N} \sum_{i=1}^{N}\left(x_{1, i}-E x_{1}\right)\left(x_{2, i}-E x_{2}\right)}{\sqrt{\frac{1}{N} \sum_{i=1}^{N}\left(x_{1, i}-E x_{1}\right)^{2}} \sqrt{\frac{1}{N} \sum_{i=1}^{N}\left(x_{2, i}-E x_{2}\right)^{2}}} \\
& =\frac{\frac{1}{N} \sum_{i=1}^{N}\left(x_{1, i}-E x_{1}\right)\left(x_{2, i}-E x_{2}\right)}{\sqrt{E\left(s_{1}^{2}\right)} \sqrt{E\left(s_{2}^{2}\right)}} \\
& =\frac{\frac{1}{N} \sum_{i=1}^{N}\left(x_{1, i}-E x_{1}\right)\left(x_{2, i}-E x_{2}\right)}{\sqrt{E\left(s_{1}^{2}\right)} \sqrt{E\left(s_{2}^{2}\right)}}
\end{aligned}
$$

Note that battery cells in actual packs are usually series-connected, so the cell terminal voltages are theoretically independent from each other, thus (A2) can be transformed into 2 separated terms (A3):

$$
\begin{gathered}
C I_{x_{1}, x_{2}}=\frac{\frac{1}{N} \sum_{i=1}^{N} x_{1, i} x_{2, i}-\frac{1}{N}\left(\sum_{i=1}^{N} x_{1, i}\right) \cdot \frac{1}{N}\left(\sum_{i=1}^{N} x_{2, i}\right)}{\sqrt{E\left(s_{1}^{2}\right)} \sqrt{E\left(s_{2}^{2}\right)}} \\
=\frac{\frac{1}{N} \sum_{i=1}^{N} x_{1, i} x_{2, i}}{\sqrt{E\left(s_{1}^{2}\right)} \sqrt{E\left(s_{2}^{2}\right)}}-\frac{\frac{1}{N}\left(\sum_{i=1}^{N} x_{1, i}\right) \cdot \frac{1}{N}\left(\sum_{i=1}^{N} x_{2, i}\right)}{\sqrt{E\left(s_{1}^{2}\right)} \sqrt{E\left(s_{2}^{2}\right)}} \\
=G D I_{C I_{1}}-G D I_{C I_{2}},
\end{gathered}
$$


where $s_{1}, s_{2}$ denote corresponding center distances of elements in vector $\left\{x_{1, i}\right\}_{i=1}^{N}$ and $\left\{x_{2, i}\right\}_{i=1}^{N}$, thus the denominators are composed of the same $s_{1}, s_{2}$, with parameters assigned $n_{s}=1, p=N^{-1}, m_{1}=m_{2}=2$.

Then we turn to investigate the numerators of the two sub-GDIs (GDI $\left.\mathrm{CI}_{1}, G D I_{C I_{2}}\right)$, the numerator of $G D I_{C I_{1}}$ is as shown in (A4):

$$
\frac{1}{N} \sum_{i=1}^{N} x_{1, i} x_{2, i}=E\left(x_{1, i} x_{2, i}\right)
$$

where $z_{C I_{1}}$ denotes the product of corresponding elements in vector $\left\{x_{1, i}\right\}_{i=1}^{N}$ and $\left\{x_{2, i}\right\}_{i=1}^{N}$, with $n_{s, 1}=1, p=N^{-1}, l_{C I_{1}}=1$; and the numerator of $G D I_{C I_{2}}$ is as shown in (A5):

$$
\frac{1}{N}\left(\sum_{i=1}^{N} x_{1, i}\right) \cdot \frac{1}{N}\left(\sum_{i=1}^{N} x_{2, i}\right)=E\left(x_{1, i}\right) E\left(x_{2, i}\right),
$$

where $z_{C I_{2}, 1}, z_{C I_{2}, 2}$ denote the corresponding elements in vector $\left\{x_{1, i}\right\}_{i=1}^{N}$ and $\left\{x_{2, i}\right\}_{i=1}^{N}$ with $n_{s, 2}=2, p=N^{-1}, l_{C I_{2}, 1}=l_{C I_{2}, 2}=1$.

So far, with respect to the feasibility of interpreting the 8th listed signal feature $C I$ by the proposed GDI formula, a strict proof by expression transformation and parameter assignment has been given.

\section{References}

1. Zhao, Y.; Liu, P.; Wang, Z.; Zhang, L.; Hong, J. Fault and defect diagnosis of battery for electric vehicles based on big data analysis methods. Appl. Energy 2017, 207, 354-362. [CrossRef]

2. Gao, Z.; Cecati, C.; Ding, S.X. A Survey of Fault Diagnosis and Fault-Tolerant Techniques—Part I: Fault Diagnosis With ModelBased and Signal-Based Approaches. IEEE Trans. Ind. Electron. 2015, 62, 3757-3767. [CrossRef]

3. Shang, Y.; Lu, G.; Kang, Y.; Zhou, Z.; Duan, B.; Zhang, C. A multi-fault diagnosis method based on modified Sample Entropy for lithium-ion battery strings. J. Power Sources 2020, 446, 227275. [CrossRef]

4. Dai, X.; Gao, Z. From Model, Signal to Knowledge: A Data-Driven Perspective of Fault Detection and Diagnosis. IEEE Trans. Ind. Inform. 2013, 9, 2226-2238. [CrossRef]

5. Zhang, C.; Jiang, Y.; Jiang, J.; Cheng, G.; Diao, W.; Zhang, W. Study on battery pack consistency evolutions and equilibrium diagnosis for serial- connected lithium-ion batteries. Appl. Energy 2017, 207, 510-519. [CrossRef]

6. Feng, X.; He, X.; Lu, L.; Ouyang, M. Analysis on the Fault Features for Internal Short Circuit Detection Using an ElectrochemicalThermal Coupled Model. J. Electrochem. Soc. 2018, 165, A155-A167. [CrossRef]

7. Chen, Z.; Xiong, R.; Lu, J.; Li, X. Temperature rise prediction of lithium-ion battery suffering external short circuit for all-climate electric vehicles application. Appl. Energy 2018, 213, 375-383. [CrossRef]

8. Hu, X.; Zhang, K.; Liu, K.; Lin, X.; Dey, S.; Onori, S. Advanced Fault Diagnosis for Lithium-Ion Battery Systems: A Review of Fault Mechanisms, Fault Features, and Diagnosis Procedures. IEEE Ind. Electron. Mag. 2020, 14, 65-91. [CrossRef]

9. Xiong, R.; Yu, Q.; Shen, W.; Lin, C.; Sun, F. A Sensor Fault Diagnosis Method for a Lithium-Ion Battery Pack in Electric Vehicles. IEEE Trans. Power Electron. 2019, 34, 9709-9718. [CrossRef]

10. Finegan, D.P.; Darst, J.; Walker, W.; Li, Q.; Yang, C.; Jervis, R.; Heenan, T.M.; Hack, J.; Thomas, J.C.; Rack, A.; et al. Modelling and experiments to identify high-risk failure scenarios for testing the safety of lithium-ion cells. J. Power Sources 2019, 417, $29-41$. [CrossRef]

11. Alavi, S.M.M.; Samadi, M.F.; Saif, M. Plating Mechanism Detection in Lithium-ion Batteries, by Using a Particle-filtering Based Estimation Technique. In Proceedings of the 2013 American Control Conference, Washington DC, USA, 17-19 June 2013; pp. 4356-4361.

12. Zhang, J.; Lee, J. A review on prognostics and health monitoring of Li-ion battery. J. Power Sources 2011, 196, 6007-6014. [CrossRef]

13. Hannan, M.A.; Lipu, M.S.H.; Hussain, A.; Mohamed, A. A review of lithium-ion battery state of charge estimation and management system in electric vehicle applications: Challenges and recommendations. Renew. Sustain. Energy Rev. 2017, 78, 834-854. [CrossRef]

14. Feng, X.; Weng, C.; Ouyang, M.; Sun, J. Online internal short circuit detection for a large format lithium ion battery. Appl. Energy 2016, 161, 168-180. [CrossRef]

15. Xu, J.; Mi, C.C.; Cao, B.; Cao, J. A new method to estimate the state of charge of lithium-ion batteries based on the battery impedance model. J. Power Sources 2013, 233, 277-284. [CrossRef]

16. Liu, K.; Hu, X.; Wei, Z.; Li, Y.; Jiang, Y. Modified Gaussian Process Regression Models for Cyclic Capacity Prediction of Lithium-Ion Batteries. IEEE Trans. Transp. Electrif. 2019, 5, 1225-1236. [CrossRef] 
17. Xiong, R.; Tian, J.; Shen, W.; Sun, F. A Novel Fractional Order Model for State of Charge Estimation in Lithium Ion Batteries. IEEE Trans. Veh. Technol. 2018, 68, 4130-4139. [CrossRef]

18. Yang, Q.; Xu, J.; Li, X.; Xu, D.; Cao, B. State-of-health estimation of lithium-ion battery based on fractional impedance model and interval capacity. Int. J. Electr. Power Energy Syst. 2020, 119, 105883. [CrossRef]

19. Zheng, Y.; Ouyang, M.; Lu, L.; Li, J.; Han, X.; Xu, L.; Ma, H.; Dollmeyer, T.A.; Freyermuth, V. Cell state-of-charge inconsistency estimation for LiFePO4 battery pack in hybrid electric vehicles using mean-difference model. Appl. Energy 2013, 111, 571-580. [CrossRef]

20. Gao, W.; Zheng, Y.; Ouyang, M.; Li, J.; Lai, X.; Hu, X. Micro-Short-Circuit Diagnosis for Series-Connected Lithium-Ion Battery Packs Using Mean-Difference Model. IEEE Trans. Ind. Electron. 2019, 66, 2132-2142. [CrossRef]

21. Zheng, Y.; Gao, W.; Ouyang, M.; Lu, L.; Zhou, L.; Han, X. State-of-charge inconsistency estimation of lithium-ion battery pack using mean-difference model and extended Kalman filter. J. Power Sources 2018, 383, 50-58. [CrossRef]

22. Cordoba-Arenas, A.; Onori, S.; Guezennec, Y.; Rizzoni, G. Capacity and power fade cycle-life model for plug-in hybrid elec-tric vehicle lithium ion battery cells containing blended spinel and layered-oxide positive electrodes. J. Power Sources 2015, 278, 473-483. [CrossRef]

23. Cong, X.; Zhang, C.; Jiang, J.; Zhang, W.; Jiang, Y. A Hybrid Method for the Prediction of the Remaining Useful Life of Lithium-Ion Batteries with Accelerated Capacity Degradation. IEEE Trans. Veh. Technol. 2020, 69, 12775-12785. [CrossRef]

24. Kong, X.; Zheng, Y.; Ouyang, M.; Li, X.; Lu, L.; Li, J.; Zhang, Z. Signal synchronization for massive data storage in modular battery management system with controller area network. Appl. Energy 2017, 197, 52-62. [CrossRef]

25. Zhao, R.; Liu, J.; Gu, J. Simulation and experimental study on lithium ion battery short circuit. Appl. Energy 2016, 173, 29-39. [CrossRef]

26. Hu, X.; Jiang, J.; Cao, D.; Egardt, B. Battery Health Prognosis for Electric Vehicles Using Sample Entropy and Sparse Bayesian Predictive Modeling. IEEE Trans. Ind. Electron. 2015, 63, 1. [CrossRef]

27. Xia, B.; Shang, Y.; Nguyen, T.; Mi, C. A correlation based fault detection method for short circuits in battery packs. J. Power Sour. 2017, 337, 1-10. [CrossRef]

28. Wang, Z.; Hong, J.; Liu, P.; Zhang, L. Voltage fault diagnosis and prognosis of battery systems based on entropy and Z -score for electric vehicles. Appl. Energy 2017, 196, 289-302. [CrossRef]

29. Li, X.; Wang, Z. A novel fault diagnosis method for lithium-Ion battery packs of electric vehicles. Measurement 2018, $116,402-411$. [CrossRef]

30. Jiang, J.; Cong, X.; Li, S.; Zhang, C.; Zhang, W.; Jiang, Y. A Hybrid Signal-Based Fault Diagnosis Method for Lithium-Ion Batteries in Electric Vehicles. IEEE Access 2021, 9, 19175-19186. [CrossRef]

31. Niri, M.F.; Dinh, T.Q.; Yu, T.F.; Marco, J.; Bui, T.M.N. State of Power Prediction for Lithium-Ion Batteries in Electric Vehicles via Wavelet-Markov Load Analysis. IEEE Trans. Intell. Transp. Syst. 2020, 1-16, 1-16. [CrossRef]

32. Zhang, M.; Jiang, Z.; Feng, K. Research on variational mode decomposition in rolling bearings fault diagnosis of the multi-stage centrifugal pump. Mech. Syst. Signal Process. 2017, 93, 460-493. [CrossRef]

33. Wang, X.; Fan, W.; Li, X.; Wang, L. Weak Degradation Characteristics Analysis of UAV Motors Based on Laplacian Eigenmaps and Variational Mode Decomposition. Sensors 2019, 19, 524. [CrossRef] [PubMed]

34. Li, Y.; Cheng, G.; Liu, C.; Chen, X. Study on planetary gear fault diagnosis based on variational mode decomposition and deep neural networks. Measurement 2018, 130, 94-104. [CrossRef]

35. Li, G.; Tang, G.; Luo, G.; Wang, H. Underdetermined blind separation of bearing faults in hyperplane space with variational mode decomposition. Mech. Syst. Signal Process. 2019, 120, 83-97. [CrossRef]

36. Dragomiretskiy, K.; Zosso, D. Variational Mode Decomposition. IEEE Trans. Signal Process. 2014, 62, 531-544. [CrossRef]

37. Xiong, J.; Zhang, Q.; Sun, G.; Zhu, X.; Liu, M.; Li, Z. An information fusion fault diagnosis method based on dimensionless in-dicators with static discounting factor and KNN. IEEE Sens. J. 2016, 16, 2060-2069. [CrossRef]

38. Hu, Q.; Si, X.-S.; Qin, A.-S.; Lv, Y.-R.; Zhang, Q.-H. Machinery Fault Diagnosis Scheme Using Redefined Dimensionless Indicators and mRMR Feature Selection. IEEE Access 2020, 8, 40313-40326. [CrossRef]

39. Xiong, J.; Zhang, Q.; Wan, J.; Liang, L.; Cheng, P.; Liang, Q. Data Fusion Method Based on Mutual Dimensionless. IEEE/ASME Trans. Mechatron. 2017, 23, 506-517. [CrossRef]

40. Chandola, V.; Banerjee, A.; Kumar, V. Anomaly Detection: A Survey. ACM Comput. Surv. 2009, 41, 15. [CrossRef]

41. Glowacz, A. Fault diagnosis of electric impact drills using thermal imaging. Measurement 2021, 171, 108815. [CrossRef] 